
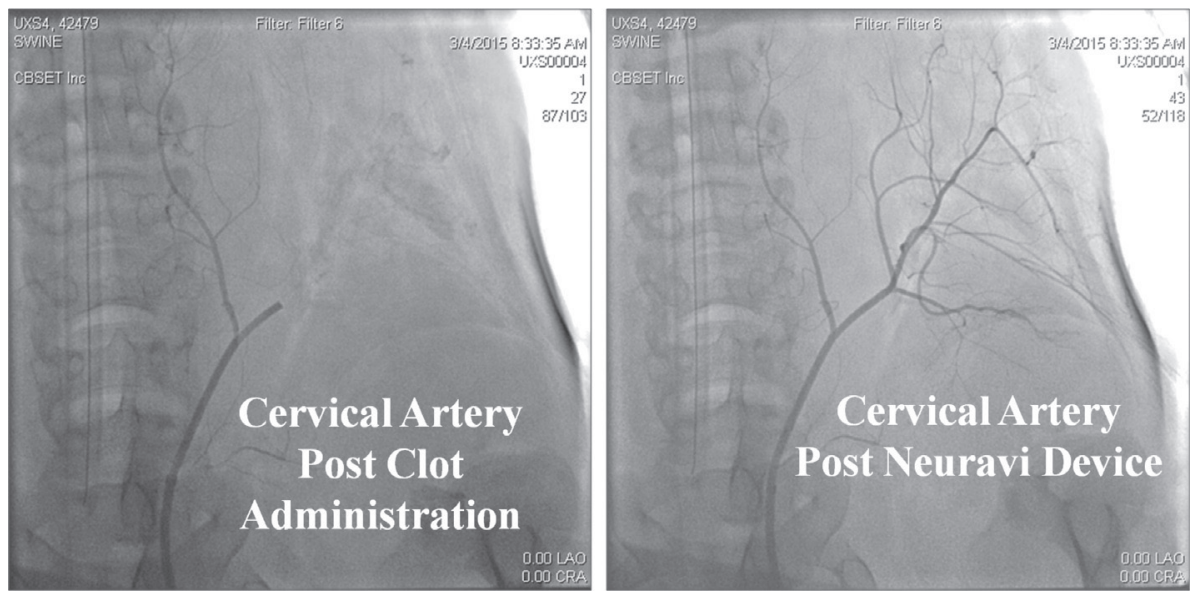

Abstract E-080 Figure 1

\section{E-080 PRECLINICAL EVALUATION OF MECHANICAL THROMBECTOMY DEVICES IN A SWINE CLOT MODEL}

${ }^{1}$ L Bailey, ${ }^{2} \mathrm{M}$ Gilvarry, ${ }^{2} \mathrm{M}$ Holian, ${ }^{1} \mathrm{~A}$ Tzafriri, ${ }^{3} \mathrm{~J}$ Stanley, ${ }^{4} \mathrm{E}$ Edelman. ${ }^{1}$ Applied Sciences, CBSET, Lexington, MA; ${ }^{2}$ Neuravi Ltd., Galway, Ireland; ${ }^{3}$ Pathology, CBSET, Lexington, MA; ${ }^{4}$ MESS MIT, Cambridge, MA

\subsection{6/neurintsurg-2016-012589.152}

Background Successful recanalization (TICI $\geq 2 \mathrm{~b}$ ) using mechanical thrombectomy (MT) with stent retriever devices is associated with good outcomes in the treatment of ischemic stroke. Preclinical TICI flow assessement is challenging due to the difficulty of consistently occluding selected blood vessels in vivo using lab-created thrombus. We describe the development of a discrete occlusive porcine clot model for predictable and repeatable assessment of TICI flow restoration and treatment effects on the vessels at the 3 day and 28 day timepoints and following MT.

Methods Firm and soft thrombus were aged for 24-48 hours prior to injection into the peripheral arteries of eight Yorkshire pigs. Oversized clots were introduced to the target region through a guide catheter via a customized luer to minimize shear/fragmentation. Clots were allowed to stabilize in the vessel prior to treatment. Clot retrieval was performed with a novel Revascularization Device (Neuravi Ltd.) or Trevo ${ }^{\circledR}$ ProVue Retriever (Stryker Inc.) as a control. TICI flow was assessed following clot administration and after thrombectomy. Vascular response to the treatment was assessed histologically at 3 and 28 days.

Results Complete occlusion was achieved in 92\% (22/24) of the targeted arteries by insertion of one (20/22) or two $(2 / 22)$ pre-measured clots. Of the occluded arteries treated, TICI 3 flow restoration was achieved in all cases (100\%) with the
Neuravi device and 90\% cases with Trevo; single pass recanalization (TICI $\geq 2$ b) rates were $92 \%$ and $80 \%$ respectively. All vessels were patent at 3 and 28 days post treatment, and histologically the prototype Neuravi revascularization device was associated with favorable local and end organ tissue responses, and was comparable to the Trevo ProVue.

Conclusions Novel methods were developed to provide consistent thrombus morphology enabling controlled delivery and adherence and facilitating comparative analysis of thrombectomy devices using angiographic (TICI) flow assessment in the preclinical swine model. Angiographic assessments and vessel response to treatment by Mechanical Thrombectomy were successfully completed following occlusion with firm and soft thrombus administration.

Disclosures L. Bailey: 1; C; Neuravi Ltd. M. Gilvarry: 5; C; Neuravi Ltd. M. Holian: 5; C; Neuravi Ltd. A. Tzafriri: 1; C; Neuravi Ltd. J. Stanley: 1; C; Neuravi Ltd.. E. Edelman: None.

\section{E-081 ASSESSMENT OF THE MAFA RATIO AS A QUANTITATIVE PROGNOSTIC MARKER OF ANEURYSM OCCLUSION AFTER FLOW DIVERTER TREATMENT}

${ }^{1} \mathrm{~V}$ Mendes Pereira, ${ }^{1} \mathrm{O}$ Brina, ${ }^{1} \mathrm{~J}$ Bracken, ${ }^{1} \mathrm{~T}$ Krings, ${ }^{2} \mathrm{~K}$ Lovblad, ${ }^{3} \mathrm{~F}$ van Nijnatten, ${ }^{4} \mathrm{D}$ Ruijters, ${ }^{4} \mathrm{~T}$ Grünhagen. ${ }^{1}$ Division of Neuroradiology - Joint Department of Medical Imaging Division of Neurosurgery - Departme, University of Toronto, Toronto, ON, Canada; ${ }^{2}$ Division of Neuroradiology - Joint Department of Medical Imaging Division of Neurosurgery Departme, University of Geneva, Geneva, Switzerland; ${ }^{3}$ Division of Neuroradiology - Joint Department of Medical Imaging Division of Neurosurgery - Departme, Philips, Best, Netherlands; ${ }^{4} I R$, Philips Healthcare, Best, Netherlands

10.1136/neurintsurg-2016-012589.153

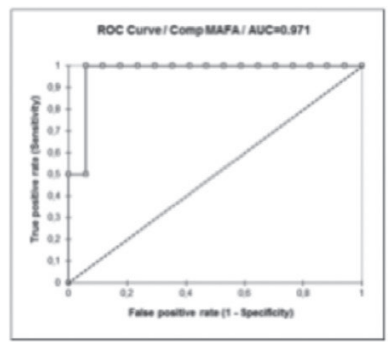

(a)

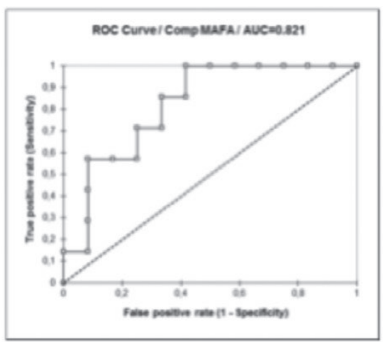

(b)

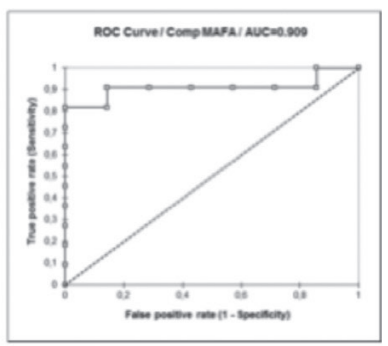

(c) 
Introduction Flow-diverter stents (FDSs) have been used effectively to treat wide neck and complex intracranial aneurysms (IAs). However, treatment strategies vary significantly. Previously, the normalized mean aneurysm flow amplitude ratio (MAFA-R) has been described as a novel metric to quantify aneurysm flow reduction that occurs as a result of FD stenting. The aim of this study was to evaluate the performance of the MAFA-R in predicting complete occlusion for IAs treated with FDSs.

Materials and methods We included patients harboring unruptured saccular IAs, which were treated with flow diverter stents. For each patient, a 3D rotational angiogram was performed prior to implantation and two high frame rate DSA sequences were acquired respectively pre- and post- FD stenting. The aneurysmal flow reduction as indicated by the MAFA-R was calculated using dedicated software (AneurysmFlow, Philips Healthcare). The MAFA-R performance for prediction of complete aneurysm occlusion was tested against the thrombosis incidence at 3, 6, and 12 months by using the receiver operating characteristic (ROC) analysis performance test. The AUC was determined, as well as the threshold maximizing the sum of sensitivity and specificity. The analysis was carried out on the total aneurysm cohort as well as for a subgroup of aneurysms $>10 \mathrm{~mm}$.

Results We included 54 consecutive patients. All cases were successfully treated with a[o1] flow diverter stent. At 3 months follow-up, 11 out of 54 aneurysms were fully occluded (20\%), and 43 displayed residual circulation (80\%). The performance of MAFA-R test for the total cohort was: AUC $=0.60, p=0.386$ and threshold $=0.62$. At 6 months $31 / 54$ aneurysms occluded (57\%), AUC $=0.657, \mathrm{p}=0.414$, threshold $=0.93$. At 12 months $39 / 52$ aneurysms occluded (75\%), $\mathrm{AUC}=0.66, \mathrm{p}=0.081$, threshold $=0.89$. For the large aneurysm subgroup, the results were: at 3 months 2/19 aneurysms occluded (11\%): AUC $=0.97, \mathrm{p}=0.014$, threshold $=0.64$; at 6 months $7 / 19$ aneurysms occluded (37\%): AUC $=0.82, \mathrm{p}<0.0001$, threshold $=0.93$; and at 12 months 11/18 aneurysms occluded (61\%): AUC $=0.91$, $\mathrm{p}<0.0001$, threshold $=0.89$.

Conclusions Aneurysm occlusion after FD stenting is determined by multiple factors including aneurysm size, morphology and the hemodynamic environment. While for the total aneurysm cohort no significance was reached, the results of this study show that for large aneurysms, the MAFA-R is an independent predictor of aneurysm occlusion at 12 months follow up (MAFA-R threshold $=0.89$ ). This real-time functional assessment may potentially help the clinician to adapt the treatment during the procedure by adding further FDS layers or intra-saccular coils. [o1]Some cases were implanted with 2 devices, BB4-4AE2-9764-89B1B81991A1\$\$

Disclosures V. Mendes Pereira: 1; C; Philips, Medtronic. O. Brina: None. J. Bracken: 5; C; Philips. T. Krings: None. K. Lovblad: None. F. van Nijnatten: 5; C; Philips. D. Ruijters: 5; C; Philips. T. Grünhagen: 5; C; Philips.

\section{E-082 VENOUS VASCULAR MALFORMATION OF THE FLOOR OF MOUTH MASQUERADING AS DERMOID TUMOR}

${ }^{1} \mathrm{~A}$ Dmytriw, ${ }^{2}$ S Song, ${ }^{3} \mathrm{P}$ Gullane, ${ }^{4} \mathrm{~K}$ terBrugge, ${ }^{5} \mathrm{E}$ Yu. ${ }^{1}$ Department of Medical Imaging, University of Toronto, Toronto, ON, Canada; ${ }^{2}$ Department of Otolaryngology, University of Toronto, Toronto, ON, Canada; ${ }^{3}$ Department of Otolaryngology, Princess Margaret Hospital, Toronto, ON, Canada; ${ }^{4}$ Department of Medical Imaging, Toronto Western Hospital, Toronto, ON, Canada; ${ }^{5}$ Department of Medical Imaging, Princess Margaret Hospital, Toronto, ON, Canada

\subsection{6/neurintsurg-2016-012589.154}

Introduction Venous vascular malformations (VVMs) are described as abnormal post-capillary lesions which exhibit low flow. These are typically malleable and may grow with endocrine fluctuations. These irregular conduits are often characterized by a soft, compressible, nonpulsatile tissue mass that may exhibit a blue tinge due to the collection of ectatic venous channels underneath the dermis. Further findings upon physical examination may include expansion of the malformation with the Valsalva maneuver, and flattening of the protrusion with applied pressure. A VVM that mimics the classic appearance of dermoid tumor on imaging has never been reported.

Case presentation We encountered a 43 year-old woman with intermittent dysphagia relating to a firm submandibular mass. Physical exam and cross-sectional imaging revealed features consistent with variant dermoid cyst. Gadolinium-enhanced MRI of the mass in T1 and T2 weighted imaging (Figure 1) revealed a lesion with T1 hypersensitivity and variable T2 signal. These results were followed-up with CT angiography which showed internal enhancement (Figure 1) possibly attributable to saponification, calcification, or rupture of the presumed dermoid cyst. However this pattern of enhancement was felt to be unusual, and digital subtraction angiography was performed to exclude a primarily vascular lesion. Catheter angiography eventually demonstrated a VVM which possessed vessels of variable size and partial thrombosis.

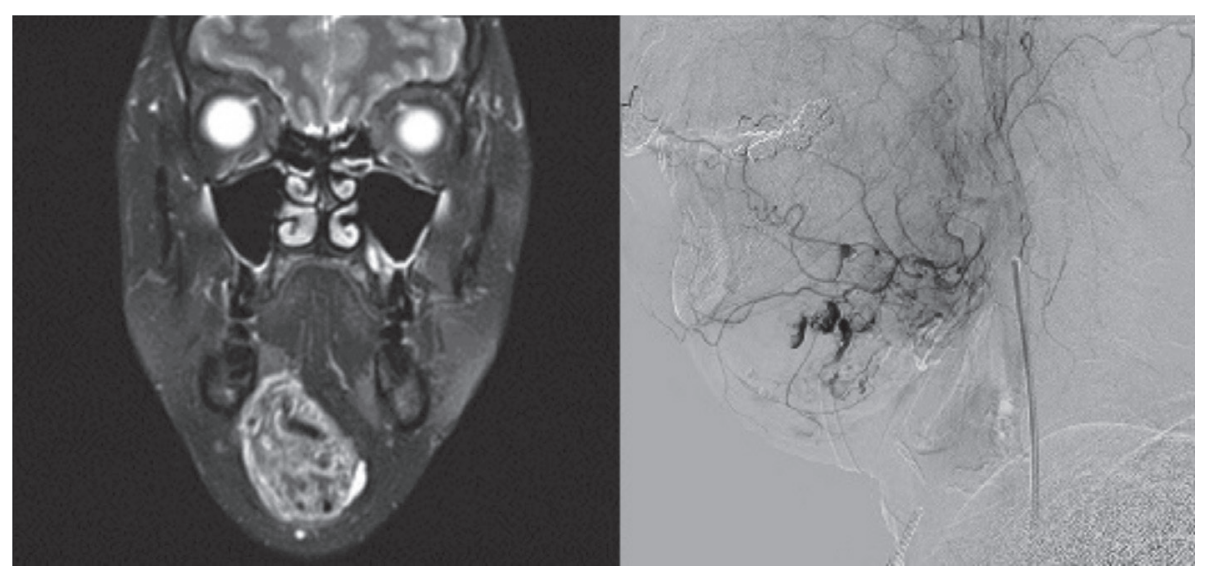

Abstract E-082 Figure 1 